\title{
Unternehmensstrategien zur Gestaltung nachhaltiger Arbeitsplätze
}

\author{
Wenn Unternehmen ambitionierte Nachhaltig- \\ keitsstrategien erfolgreich umsetzen wollen, sind \\ sie auf die Mitwirkung und das Engagement der \\ Beschäftigten angewiesen. Welche Instrumente \\ können dazu beitragen, die Mitarbeitenden zu \\ einem Engagement zu motivieren? \\ Von Martina Schäfer und Elisabeth Süßbauer
}

\section{Einleitung}

In den letzten Jahren hat die aktive Rolle der Mitarbeitenden für die Umsetzung von Nachhaltigkeitsstrategien in Unternehmen an Bedeutung gewonnen (Lozano 2015). Dabei können verschiedene Diskussionsstränge unterschieden werden. Ein erster Strang fokussiert auf den Einbezug von Nutzer/innen-Wissen in die Gestaltung und Verbesserung von Produkten und Dienstleistungen wie zum Beispiel Öko-Innovationen (z. B. Buhl et al. 2016). Ein zweiter Strang beschäftigt sich damit, wie das Erfahrungswissen der Mitarbeitenden genutzt werden kann, um unternehmerische Prozesse in Richtung Nachhaltigkeit zu verbessern (z. B. Vermeidung von Materialverschwendung und Emissionen) (Wolf 2013). Ein dritter Ansatz zielt darauf ab, die Mitarbeitenden für nachhaltiges Alltagshandeln am Arbeitsplatz bzw. Strategien für nachhaltige Arbeitsplätze zu motivieren (Muster 2011; Süßbauer/Schäfer 2018, 2019).

Obwohl bereits Konzepte zur Verbindung von Mitarbeitenden-Engagement und der nachhaltigen Gestaltung des Arbeitsplatzes vorliegen (z. B. green work-life balance, Muster et al. 2011), fehlen bisher empirische Befunde $\mathrm{zu}$ entsprechenden unternehmerischen Strategien. Die vorhandenen Studien fokussieren entweder auf individuelle oder organisationale Faktoren (Lo et al. 2012). Die hier vorgestellte Studie hat im Rahmen des Forschungsprojekts IMKoN - Integration von Mitarbeitern als Konsumenten in Nachhaltigkeitsinnovationsprozesse Strategien von acht Unternehmen dahingehend untersucht, inwieweit sie Anreize für eine Veränderung der Konsumroutinen der Mitarbeitenden in Richtung Nachhaltigkeit schaffen und sie gleichzeitig in eine entsprechende Gestaltung des Arbeitsplatzes einbeziehen. Dabei verstehen wir Unternehmen (oder andere Organisationen, die Arbeitsplätze bereitstellen) als Settings, die nachhaltiges Alltagshandeln entweder eher unterstützen oder behindern können.

\section{Konzeptioneller Hintergrund}

Wir verwenden einen konzeptionellen Rahmen, der von praxistheoretischen Ansätzen sowie Konzepten organisationalen Lernens inspiriert ist. In Ergänzung zu den meisten anderen Studien verbindet dieser Rahmen, in Anlehnung an das Verständnis von Arbeitsplätzen als „Setting“, strukturelle Elemente mit Aspekten individuellen Handelns.

Um den Gewohnheitscharakter von Konsumhandeln am Arbeitsplatz zu berücksichtigen, greifen wir auf praxistheoretische Ansätze zurück. Soziale Praktiken werden als routinierte Arten von Verhalten charakterisiert, die von Personengruppen als Teil ihres täglichen Lebens regelmäßig ausgeführt werden (Reckwitz 2002; Verbeek et al. 2008). Die Stabilität von sozialen Praktiken hängt nach Reckwitz (2002) insbesondere von der engen Verbindung zwischen drei Elementen - Objekte, Bedeutung und Kompetenzen - ab. Um kontinuierlich ausgeführt zu werden, benötigen Praktiken sowohl Objekte, Infrastrukturen, Werkzeuge (= Dinge), als auch mentale Aktivitäten, Emotionen und Motivation (= Bedeutung) und Fähigkeiten sowie das geteilte Verständnis, wie bestimmte Dinge getan werden (= praktische Kompetenzen) (Shove et al. 2012). Obwohl die gewohnheitsmäßige Ausübung sozialer Praktiken mit ihrer häufigen Wiederholung zusammenhängt, können sich Praktiken mit der Zeit verändern (Warde 2005). Shove et al. (2012) beschreiben verschiedene Stadien im Leben einer Praxis, je nachdem, ob die Verbindungen zwischen den drei Elementen noch nicht hergestellt sind (Proto-Praktiken), vorhanden sind (ausgeübte Praktiken) oder nicht mehr bestehen (Ex-Praktiken).

Von Vorarbeiten, die sich mit organisationalem Lernen befassen, greifen wir zum einen die Kritik an der Annahme auf, dass Nachhaltigkeitshandeln sich „automatisch“ in Form einer Kaskade von der Leitung in alle hierarchischen Stufen des Unternehmens ausbreitet (Robertson et al. 2013). Harris und Crane (2002) haben dagegen beobachtet, dass Unternehmen relativ unverändert durch formale Strategien bleiben, wenn keine komplementäre unterstützende informelle Kultur existiert. Sie empfehlen daher, sich nicht auf die „offizielle“ Organisationskultur zu konzentrieren, sondern auch Routinen, Strukturen und Strategien als Ausdruck der gelebten Unternehmenskultur in den Blick zu nehmen. Damit einher gehen Befunde bezüglich der Notwendigkeit, die Bedürfnisse und Erfahrungen der Mitarbeitenden in die Gestaltung des Arbeitsplatzes einzubeziehen (Blazejewski et al. 2018; Heisserer 2013; Klade et al. 2013; Schultz et al. 2010; Nye et al. 2010). Mit der Verwendung praxistheoretischer Ansätze begegnen wir außer- 


\begin{tabular}{|c|c|c|c|}
\hline \multirow{4}{*}{$\begin{array}{l}\text { Kategorien } \\
\text { 1. Unterstützende Strukturen schaffen: } \\
\text { Bereitstellen von Elementen für neue } \\
\text { (nachhaltigere) Praktiken }\end{array}$} & \multicolumn{3}{|c|}{ Ermöglichende Strukturen und Aktivitäten } \\
\hline & Dinge & $\begin{array}{l}\text { Gestaltung von Gebäude, Büros, Freiflächen } \\
\text { (Infrastruktur, Ausstattung, finanzielle Anreize) }\end{array}$ & \multirow{6}{*}{$\begin{array}{l}\text { 4. Mitarbeitenden-Beteiligung } \\
\text { I formal: Ideen-Management } \\
\text { I informell: Partizipative Strukturen }\end{array}$} \\
\hline & Bedeutung & $\begin{array}{l}\text { Unternehmenskultur } \\
\text { I explizit: Vision, Mission, Regeln, Code of Conduct } \\
\text { I implizit: Soziale Normen und Werte }\end{array}$ & \\
\hline & Kompetenzen & $\begin{array}{l}\text { Kompetenzen } \\
\text { I formal: Training, Weiterbildung } \\
\text { I informell: inspirierende Lernumgebung }\end{array}$ & \\
\hline $\begin{array}{l}\text { 2. Experimentieren ermöglichen: } \\
\text { Knüpfen von Verbindungen zwischen } \\
\text { Elementen neuer Praktiken }\end{array}$ & \multicolumn{2}{|c|}{$\begin{array}{l}\text { Soziale Aktivitäten, um nachhaltigere Praktiken erproben zu können } \\
\text { einzelne Aktivitäten/Initiativen } \\
\text { reguläre Kampagnen }\end{array}$} & \\
\hline 3. Zur Stabilisierung von neuen & \multirow{2}{*}{\multicolumn{2}{|c|}{$\begin{array}{l}\text { Unterstützung nachhaltigen Konsums in verschiedenen Settings } \\
\text { Maßnahmen und Angebote, die Praktiken am Arbeitsplatz mit denen } \\
\text { im Privatleben verbinden }\end{array}$}} & \\
\hline Praktiken beitragen & & & \\
\hline
\end{tabular}

Tabelle 1: Konzeptioneller Rahmen

(Quelle: nach Süßbauer et al. 2018)

dem dem von einigen Autor/innen formulierten Defizit, dass die materiellen Bedingungen und Strukturen selten in empirischen Studien zu umweltbewusstem Handeln am Arbeitsplatz berücksichtigt werden (Lo et al. 2012; Ones et al. 2012).

Für eine integrative Analyse von Unternehmensstrategien für nachhaltige Arbeitsplätze haben wir einen konzeptionellen Rahmen entwickelt, der Unternehmen als Settings versteht, die Mitarbeitende in Richtung nachhaltigerem Alltags- oder Konsumhandeln motivieren können. Dabei besteht der Vorteil von Unternehmen darin, dass sie - im Gegensatz zu anderen Akteuren (wie NGOs oder Kommunen) alle Praktikenelemente Objekte, Bedeutung und Kompetenzen - sowie die verschiedenen Stadien der Entwicklung und Stabilisierung neuer Praktiken adressieren können. Tabelle 1 gibt einen Überblick über den von uns entwickelten konzeptionellen Untersuchungsrahmen.

\subsection{Unterstützende Strukturen schaffen}

Angelehnt an praxistheoretische Überlegungen sollten sich Nachhaltigkeitsziele und -aktivitäten in den materiellen und organisationalen Strukturen niederschlagen, wobei es sinnvoll ist, alle drei Praktikenelemente zu berücksichtigen (Süßbauer et al. 2018). Dies sind zum einen unterstützende materielle Strukturen wie zum Beispiel der Bezug von Ökostrom, das Angebot von vegetarischen Gerichten in der Kantine oder die umweltfreundliche Beschaffung von Büromaterial. Zum anderen sollte sich Nachhaltigkeit auf der Bedeutungsebene als integraler Teil der Unternehmenskultur, zum Beispiel in der formulierten Vision, formal festgelegten und informellen Regeln, Anerkennungsmechanismen sowie der Vorbildfunktion der Führungsebene niederschlagen. Drittens können Kompetenzen für nachhaltiges Alltagshandeln über Informationsvermittlung, Weiterbildungen oder - stärker informell - Möglichkeiten des Austauschs und eine inspirierende Lernumgebung vermittelt werden (Holweg et al. 2018; Schultz et al. 2010).

\subsection{Experimentieren ermöglichen}

Unterstützende strukturelle Voraussetzungen zu schaffen, ist ein wichtiger Schritt, garantiert aber nicht, dass Mitarbeitende ihre Routinen verändern. Um Veränderungen zu beför- dern, können Unternehmen über Kampagnen, Aktionen, Anreize, Wettbewerbe et cetera zum Experimentieren mit neuen Praktiken anregen. Möglichkeiten des informellen Austauschs über die gewonnenen Erfahrungen können die Wirkungen experimentellen Handelns verstärken.

\subsection{Zur Stabilisierung neuer Praktiken beitragen}

Experimentieren kann wichtige Impulse für Veränderungen des Alltagsverhaltens geben, letztlich kommt es aber auf die dauerhafte Integration der veränderten Verhaltensweisen in alltägliche Routinen an. Die Stabilisierung neuer Praktiken kann nur in längeren Zeiträumen durch ihre Wiederholung und die Verknüpfung mit anderen Praktiken vonstattengehen. Unternehmen könnten hierzu nur bedingt beitragen, indem sie zum Beispiel Praktiken aus verschiedenen Feldern adressieren (z. B. Einkaufen und Mobilität zur Arbeit) oder Angebote machen, die Praktiken am Arbeitsplatz mit privaten Praktiken verbinden (z. B. Finanzierung der Bahn Card für dienstliche und private Fahrten).

\subsection{Mitarbeitendenbeteiligung}

Mitarbeitende sollten auf allen Ebenen der Gestaltung des Arbeitsplatzes und der Planung und Durchführung von Aktionen zur Gestaltung nachhaltiger Arbeitsplätze über informelle Formate und formalisierte Instrumente eingebunden werden, um ihre Bedürfnisse zu berücksichtigen und die Motivation zur Teilnahme zu erhöhen.

Der aus der Literatur abgeleitete konzeptionelle Untersuchungsrahmen diente im Rahmen des Projekts als Grundlage, um die unternehmerischen Strategien für nachhaltige Arbeitsplätze vergleichend zu untersuchen.

\section{Methodisches Vorgehen}

Zur Untersuchung, ob und wie alltägliche Konsumerfahrungen von Beschäftigten in Innovationsprozesse eingebunden werden können, wurden in IMKoN Daten aus acht deutschen Unternehmen ausgewertet. Um eine möglichst große Bandbreite an organisationalen Strukturen und Kulturen zu 
untersuchen, wurden insgesamt acht Unternehmen aus unterschiedlichen Branchen im Business-to-Consumer-Bereich ausgewählt, darunter zum Beispiel Wasch- und Reinigungsmittel, Haushaltsgeräte, Versandhandel, Energieversorgung und Print- und Onlinemedien. Vertreten waren drei Großunternehmen mit 2.000 bis 5.500 Beschäftigten (Unternehmen A-D) und fünf Ökopionier-Unternehmen mit 65 bis 100 Beschäftigten am jeweiligen Hauptstandort (Unternehmen E-H). Durch die geringe Fallzahl handelt es sich im Hinblick auf die Typenbildung um eine explorative Untersuchung.

Um ein umfassendes Bild von den Unternehmen zu erhalten, haben wir verschiedene Methoden und Perspektiven kombiniert. Die Analysen umfassten Fragen nach den unternehmerischen Strukturen und Aktivitäten im Hinblick auf die Gestaltung nachhaltiger Arbeitsplätze und hinsichtlich des Einbezugs der Mitarbeitenden in die Gestaltung des Arbeitsplatzes.

Zunächst führten wir leitfadengestützte Interviews mit jeweils drei bis fünf Mitarbeitenden des Innovations-, Nachhaltigkeits-, Personal- und Gebäudemanagements durch. In den kleineren Unternehmen wurde auch die Geschäftsführung befragt. Die Interviews dauerten circa 60 Minuten. Sie wurden transkribiert und inhaltsanalytisch analysiert. In Kombination mit Informationen aus offiziellen Berichten und der Webseite, repräsentieren die Interviews mit dem Management für uns die offizielle Unternehmenskultur der Fallstudien.

Weiterhin führten wir in allen Unternehmen (außer Unternehmen A) Fokusgruppen mit fünf bis zehn Mitarbeitenden durch, um mehr über die Alltagsroutinen und die gelebte Unternehmenskultur im Hinblick auf Nachhaltigkeit und Partizipation zu erfahren. Dabei wurden Mitarbeitende aus verschiedenen Abteilungen und Hierarchiestufen beteiligt. Die Fokusgruppen dauerten 180 Minuten. Die Audioaufzeichnungen wurden transkribiert und inhaltsanalytisch ausgewertet.

Schließlich können wir für die Fragestellung dieses Artikels ergänzend die Antworten von zwei Fragen einer quantitativen Befragung mit Mitarbeitenden der Fallstudien-Unternehmen verwenden. Sechs der acht Unternehmen (alle außer Unternehmen A und C) nahmen an der digitalen Befragung teil. Von insgesamt 4.870 adressierten Mitarbeitenden wurden von 840 Antworten erhalten. 616 Fragebögen konnten für die Analyse verwendet werden. Die hier verwendeten Antworten beziehen sich auf die Zufriedenheit mit dem Arbeitsplatz.

Basierend auf der inhaltsanalytischen Auswertung der Interviews und Fokusgruppen nach den im Untersuchungsrahmen aufgeführten Kategorien sowie den Ergebnissen der quantitativen Befragung, verfassten wir zunächst individuelle Fallstudienbeschreibungen jedes Unternehmens. In Anlehnung an die Methode der qualitativen Typenbildung (Kluge 2000), wurden die Fallstudien in einem zweiten Schritt systematisch nach Ähnlichkeiten und Unterschieden verglichen. Dieser qualitative Vergleich führte zur Identifikation von verschiedenen Typen unternehmerischer Strategien zur Gestaltung nachhaltiger Arbeitsplätze.

\section{Ergebnisse}

Für die erwähnte Typenbildung unterscheiden wir hauptsächlich zwei Dimensionen, die relevant sind, um Unterschiede und Gemeinsamkeiten der unternehmerischen Strategien zu identifizieren: A) Unterstützende Strukturen und Aktivitäten zur Gestaltung nachhaltiger Arbeitsplätze und B) Mitarbeitendenbeteiligung. In den folgenden Absätzen werden diese Dimensionen anhand von Ergebnissen aus den empirischen Untersuchungen dargestellt, gefolgt von der qualitativen Typenbildung.

\subsection{Strukturen und Aktivitäten}

In den untersuchten Unternehmen können verschiedene Niveaus der Bereitstellung unterstützender Strukturen und Aktivitäten identifiziert werden, die wir in „kohärent“ und „fragmentiert" unterscheiden.

Kohärente Strukturen und Aktivitäten zeichnen sich dadurch aus, dass die Unternehmen im Hinblick auf alle drei Kategorien der Entwicklung und Stabilisierung neuer Praktiken aktiv sind und alle drei Praktikenelemente adressieren (Tabelle 1).

In der Gruppe der untersuchten Unternehmen wiesen die Unternehmen D, G und H kohärente Unterstützungsstrukturen in allen Konsumbereichen (Ressourcen- und Energienutzung, Ernährung, Mobilität) auf und fielen durch konsequente Optimierungsstrategien auf. Dies spiegelte sich auch in den Ergebnissen der Fokusgruppen wider, in denen die Teilnehmenden angaben, dass nachhaltiges Alltagshandeln am Arbeitsplatz Teil der gelebten Unternehmenskultur ist. In der quantitativen Befragung äußerten 44-51\% der Befragten, dass sie mit der Unterstützung für nachhaltiges Alltagshandeln am Arbeitsplatz zufrieden sind. Die nachhaltige Ausstattung in diesen Unternehmen betrifft viele Bereiche, wie zum Beispiel umweltfreundliche Beschaffung, die Erzeugung erneuerbarer Energie, Trinkwasserspender, E-Bikes für die unternehmensinterne Mobilität, Duschen für Fahrradfahrer und Parkplätze für Carsharing-Autos. Teilweise werden finanzielle Anreize für die Nutzung des öffentlichen Nahverkehrs oder das Ausleihen von Fahrrädern für alle Mitarbeitenden angeboten.

Auf der Bedeutungsebene nimmt die Geschäftsführung neben dem Vertreten eines Leitbilds von "starker Nachhaltigkeit“ häufig auch im praktischen Handeln eine Vorbildunktion ein.

„Die beiden Geschäftsführer sind der Inbegriff von nachhalti-

gem Leben und leben das selber ganz, ganz stark vor [...]."

Weiterhin sorgen explizite Regeln, die regelmäßig in schriftlicher und/oder mündlicher Form kommuniziert werden (z. B. Code of Conduct), dafür, dass die Ausübung nachhaltiger Praktiken befördert wird. Beispielsweise gilt in einem der Unternehmen die Regel, dass Dienstreisen im Inland nicht auf dem Luftweg durchgeführt werden. Dies und der informelle Austausch zu Themen dieser Art hat zur Folge, dass nachhaltiges Alltagshandeln im Unternehmen als "normal“ angesehen wird. 
„Also, wir können unsere Kollegen aufmerksam machen auf ihr Verhalten - heizen, Fenster öffnen, Stoßlüftung und so. Das ist was, wo wirklich jeder darauf achtet."

Die Gruppe dieser Unternehmen bietet regelmäßig Möglichkeiten an, Erfahrungen mit neuen Praktiken zu sammeln, wie zum Beispiel einen jährlichen Fahrradwettbewerb. Die Aktionen sind häufig längerfristig angelegt, teamorientiert, beinhalten sozialen Austausch und werden durch finanzielle Anreize oder digitale Tools unterstützt, um die Integration in den Alltag zu gewährleisten. In den Fokusgruppen wurde deutlich, dass einige Mitarbeitende Anregungen aus diesen Aktionen mitgenommen und nachhaltigeres Alltagshandeln verstetigt haben. Auch wenn manche Unternehmen nicht erfolgreich damit sind, neue organisationale Routinen zu etablieren (z. B. Einführung eines „Veggie Days“), wird über diese Themen viel diskutiert.

Für die Stabilisierung nachhaltiger Praktiken machen diese Unternehmen Angebote, die das Alltagshandeln am Arbeitsplatz mit dem im privaten Umfeld verbinden, wie zum Beispiel die Möglichkeit, regionales Biofleisch zu bestellen oder die firmenfinanzierte BahnCard auch privat zu nutzen. Weiterhin werden die Mitarbeitenden darin unterstützt, ihre privaten Nachhaltigkeitserfahrungen und -ideen in das Unternehmen einzubringen. So äußerten sich Mitarbeitende sehr positiv zu den Anregungen aus dem Unternehmen für ihre privaten Alltagsgewohnheiten.

„Also man wird echt viel inspiriert auch für sein Zuhause.

Durch den Austausch, der erwünscht ist, kriegt man immer

mehr Ideen. Und die ganzen Aktionen - also ich finde

es unglaublich, was ich da schon alles mitgenommen habe."

Die Mehrzahl der Unternehmen zeichnet sich eher durch fragmentierte unterstützende Strukturen und Aktivitäten zur Gestaltung nachhaltiger Arbeitsplätze aus. Im Unterschied zu den kohärenten Unterstützungsstrukturen sind dies Maßnahmen in einzelnen Bereichen oder temporärer Natur. Dies war der Fall bei allen untersuchten Großunternehmen und bei zwei der kleinen und mittelständischen Unternehmen (KMU). In der quantitativen Befragung waren nur 15 bis $23 \%$ der Mitarbeitenden von Unternehmen dieser Gruppe zufrieden mit der Unterstützung für nachhaltiges Alltagshandeln am Arbeitsplatz.

Auf der Ebene der materiellen Strukturen überwiegen in diesen Unternehmen häufig Ausstattungen wie Regenwassernutzung oder der Bezug von Ökostrom, die wenig Einfluss auf die täglichen Routinen der Mitarbeitenden haben. In manchen Fällen existieren nachhaltige Angebote, wie beispielsweise ein "Klimateller" in der Kantine, die aber nicht ausreichend kommuniziert werden. Dazu kommt, dass die Default-Einstellungen nachhaltiges Handeln teilweise nicht unterstützen (wie z. B. 1-seitiger Druck, Vorzug für Flüge beim internen Buchungssystem für Dienstreisen etc.).

Auf der Ebene der Bedeutung ist das Nachhaltigkeitsverständnis häufig reduziert auf Aspekte des Umweltmanagements. Der Fokus der Nachhaltigkeitsaktivitäten liegt daher

\section{„Bisher fehlen empirische Befunde zu unternehmerischen Strategien für die nachhaltige Gestaltung des Arbeitsplatzes."}

auf dem Kerngeschäft, während die Potenziale für nachhaltiges Alltagshandeln am Arbeitsplatz eher als nicht bedeutsam wahrgenommen werden. Weiterhin fungiert das Führungspersonal in dieser Gruppe von Unternehmen teilweise nur begrenzt als Vorbild.

„Wobei unser CEO mit gutem Beispiel vorangeht, der fährt

auch Elektroauto. [...] Gleichzeitig hat er aber auch einen

Maserati zu Hause. Es ist seine private Angelegenheit, aber was die Firma betrifft, ist er da sehr vorbildlich."

Regeln, die nachhaltige Praktiken unterstützen, sind in dieser Gruppe der Unternehmen eher selten oder werden ungenügend kommuniziert. Informelle Regeln, wie zum Beispiel, dass Vorschläge ausgedruckt werden müssen, damit sie auf Treffen diskutiert werden können, machen deutlich, dass nachhaltiges Alltagshandeln nicht Teil der gelebten Unternehmenskultur ist. Dies macht es für die engagierteren Mitarbeitenden auch schwerer, derartige Themen auf einer informellen Ebene anzusprechen. Übereinstimmend mit der quantitativen Befragung sehen die Mitarbeitenden dieser Gruppe von Unternehmen noch Potenziale hinsichtlich unterstützender Strukturen für nachhaltiges Alltagshandeln am Arbeitsplatz.

„Unter den Beschäftigten sollte das Bewusstsein für Nachhaltigkeit wirklich gestärkt werden [...]. Da fällt mir viel zu ein, wo das Unternehmen wirklich mehr Anregungen geben könnte."

In dieser Gruppe von Unternehmen hat die Vermittlung von Kompetenzen für nachhaltiges Alltagshandeln eher einen geringen Stellenwert. Aktivitäten zur Erprobung neuer Praktiken werden häufig nur einmalig oder kurzzeitig angeboten. Ihr Schwerpunkt liegt oft in der Stärkung des Bewusstseins für klimafreundlichen Konsum allgemein, wie zum Beispiel der Durchführung von Nachhaltigkeitstagen oder dem Pflanzen von Bäumen auf den Grünflächen des Unternehmens. Gerade in den Großunternehmen geben die Teilnehmenden der Fokusgruppen an, dass Aktivitäten dieser Art häufig nur von einer kleinen Gruppe der Beschäftigten wahrgenommen werden und die Wirkungen eher kurzzeitiger Natur sind.

„Es gab schon immer mal wieder so eine Aktion, wo es auch ums Sparen im Büro ging, also Papier einsparen und sowas. Das ist dann von kurzer Dauer, manche nehmen das gar nicht so richtig wahr und das ist ihnen egal und das war es dann." 


\section{„Wenn Unternehmen eine nachhaltige Arbeitsplatzgestaltung kontinuierlich unterstützen, sind die Beschäftigten zufriedener."}

\subsection{Beteiligung der Mitarbeitenden}

Die zweite zentrale Dimension, die wir untersucht haben, sind Strukturen und Aktivitäten im Bereich der Beteiligung der Mitarbeitenden, die sowohl formalisiert sein können (z. B. Ideen-Management) oder eher einen informellen Charakter haben können (z. B. Unterstützung bei der Umsetzung von Ideen). Auf Basis der empirischen Untersuchungen konnten wir drei Ausprägungen dieser Dimension identifizieren: paternalistisch, durchlässig und partizipativ.

Alle Großunternehmen sowie eines der KMU verfolgen eher einen paternalistischen Stil des Einbezugs der Mitarbeitenden. Sie bieten teilweise formalisierte Möglichkeiten, wie einen „grünen Briefkasten“, digitale Instrumente oder formale Treffen an, um Ideen einzubringen. In den Fokusgruppen wurde deutlich, dass häufig nicht gewährleistet ist, dass die Mitarbeitenden Feedback zu ihren Vorschlägen bekommen oder Erklärungen, warum ihre Ideen nicht für eine Umsetzung berücksichtigt wurden. In Übereinstimmung damit gaben in der quantitativen Befragung nur 4-7\% der Teilnehmenden an, dass sie das Gefühl haben, ihre Vorschläge werden wertgeschätzt. Das paternalistische Vorgehen ist durch eine hierarchische Entscheidungskultur gekennzeichnet, die für Mitarbeitende, die neue Ideen einbringen und ihre Umsetzung vorantreiben wollen, eine Barriere darstellt. Insbesondere in den größeren Unternehmen wurde erwähnt, dass die Beschäftigung mit Themen jenseits des Kerngeschäfts vom Management teilweise nicht erwünscht ist.

„Aber irgendwie besteht eher so eine Angst vor Kontrollverlust oder: ,Moment, der hat Zeit, sich mit organisatorischen Fragen zu beschäftigen?' [Finger schnipsen] Dann kriegt man einen Bereich zusätzlich! Und man kann das erstmal abarbeiten, weil man hat ja offensichtlich Zeit, sich um Dinge zu kümmern, die nicht zu den Kernaufgaben gehören. “

Die Mitarbeitenden haben den Eindruck, dass es für sie nicht möglich ist, Entscheidungsträger zu adressieren, was zu Frustration führt. Insbesondere jüngere Mitarbeitende, die das Unternehmen wegen seiner Nachhaltigkeitsorientierung ausgewählt haben, sind enttäuscht, dass sie wenig Möglichkeiten haben, eigene Ideen einzubringen, und dass Veränderungen nur mit großem persönlichem Aufwand möglich erscheinen.

„Ich glaube, man muss sich irgendwie entscheiden, da mitzu-

laufen, ja, und einfach geschehen zu lassen. Oder zu sagen:
,Ich stehe zu meinen Gedanken und zu meinen Ideen. 'Und dann muss man tatsächlich sehr, sehr viel Eigeninitiative mitbringen. Man muss sich durchboxen und man weiß oft nicht, was oben passiert."

Zwei der KMU zeigten eine andere Art der Organisationskultur, die wir als „durchlässig“ bezeichnen. Diese Unternehmen haben auf der einen Seite keine formalisierten Instrumente des Ideen-Managements, was gegebenenfalls dazu führen kann, dass Potenziale verloren gehen. Auf der anderen Seite sind sie durch flache Hierarchien und eine Kultur der „offenen Türen“ charakterisiert. In den Fokusgruppen wurde deutlich, dass die Mitarbeitenden einen unkomplizierten Zugang zu Entscheidungsträger/innen haben und ihre Ideen einbringen können, insbesondere was Verbesserungen in ihren professionellen $\mathrm{Zu}$ ständigkeiten angeht. In der quantitativen Befragung haben die Mitarbeitenden zu 11 und zu 34\% geäußert, dass sie das Gefühl haben, ihre Anregungen werden wertgeschätzt. Die höhere Zufriedenheit wurde in dem Unternehmen erzielt, dass kohärente Unterstützungsstrukturen aufweist.

„Mir wird da hier zum Beispiel auch freie Hand gelassen, so zu arbeiten, wie ich das zu Hause auch machen würde. Das heißt, ich habe eine Idee, dann kann ich einfach auf dem kurzen Dienstweg die Sachen so schnell umsetzen."

Im Hinblick auf die Gestaltung nachhaltiger Arbeitsplätze gibt es eine generelle Offenheit, aber den Mitarbeitenden werden nicht explizit Zeitressourcen zur Verfügung gestellt, um ihre Ideen weiterzuentwickeln und umzusetzen. Sie fühlen sich eher selbst verantwortlich für die Organisation der Umsetzung, gegebenenfalls in ihrer freien Zeit. Diese Ambivalenz spiegelt sich auch in den Äußerungen der Mitarbeitenden wider:

„Es ist nicht so, dass eine Idee irgendwo eingeworfen wird und dann nimmt sich dem jemand an, sondern man muss dann selber auch daran arbeiten."

Eine eher partizipative Unternehmenskultur fand sich in zwei der KMU, die beide Instrumente anbieten, um Ideen einzuspeisen, wie zum Beispiel reguläre Sitzungen für Mitarbeitende aller Hierarchiestufen. Das Ideen-Management in diesen Unternehmen umfasst konstruktives Feedback: Jede/r Mitarbeitende erhält in einer festgelegten Zeitspanne eine Rückmeldung bezüglich der Umsetzungsmöglichkeiten der eingebrachten Ideen.

„Und das ist schon schön, weil alles, was so eingeht, wird echt genau geprüft, ob man es irgendwie umsetzen kann. Und nur wenn es irgendwie gar nicht geht, dann geht es halt nicht.

Aber ich habe schon das Gefühl, dass da sehr viel umgesetzt wird von den Ideen der Mitarbeitenden."

Weiterhin fanden wir drei formale Instrumente, die es den Mitarbeitenden ermöglichen, ihre Ideen einzubringen: a) die Verfügbarkeit über ein bestimmtes zeitliches Budget für Nachhaltigkeitsengagement, b) die Möglichkeit, in einer Arbeitsgruppe zur nachhaltigen Gestaltung des Arbeitsplatzes mitzuwirken und c) die Benennung eines Ansprechpartners für Umweltthemen, der zwischen den normalen Mitarbeitenden und dem Management vermittelt. 
"Ich finde, was gut ist, dass die beiden Umweltbeauftragten sehr präsent sind [...]. Das heißt, man kennt sie und man kann sich mit allem immer jeder Zeit an sie wenden. Also von daher gibt es sehr gut die Möglichkeit, da seine Ideen unterzubringen." Diese formalen Instrumente werden kombiniert mit einer dialogischen Unternehmenskultur, die die Mitarbeitenden als „ganze Menschen“ über ihre Rolle als Mitarbeitende hinaus wertschätzt. Es existieren sowohl eine generelle Offenheit als auch Möglichkeiten des informellen Austauschs unter den Beschäftigten. Die Zufriedenheit mit der Wertschätzung ihrer Ideen ist bei den Mitarbeitenden dieser Unternehmen mit 24 und $44 \%$ relativ hoch.

\subsection{Typenbildung}

Durch die Kombination der vorher beschriebenen Dimensionen und ihrer Unterkategorien gelangen wir zu vier „Idealtypen“ von unternehmerischen Strategien zur Gestaltung nachhaltiger Arbeitsplätze: formalisiert, ganzheitlich, abwartend und experimentell (Abbildung 1).

Im Folgenden beschreiben wir die identifizierten Typen kurz und ordnen die untersuchten Fall-Unternehmen grob den jeweiligen Typen zu.

Typ 1: Unternehmen des Typs formalisierte Unternehmensstrategie stellen professionelles Ideen-Management und umfangreiche unterstützende Strukturen und Aktivitäten für nachhaltiges Alltagshandeln am Arbeitsplatz zur Verfügung. Sie sind jedoch charakterisiert durch eher hierarchische Unterscheidungsstrukturen und eine Top-down-Strategie.

Typ 2: Unternehmen, die eine ganzheitliche Unternehmensstrategie verfolgen, bemühen sich um vielfältige Möglichkeiten der Mitarbeitendenbeteiligung. Sie sind charakterisiert durch eine dialogische Unternehmenskultur, die offen ist für eine Diversität von Praktiken. Wegen der Kombination formaler und informeller Partizipationsformate und der Bereitstellung kohärenter Unterstützungsstrukturen sind nachhaltige Alltagspraktiken Teil der gelebten Unternehmenskultur. Von den untersuchten Unternehmen konnten zwei KMU diesem Typ zugeordnet werden.

Typ 3: In Unternehmen mit einer abwartenden Unternehmensstrategie finden wir die Kombination von fragmentierten Unterstützungsstrukturen für nachhaltiges Alltagshandeln mit geringen Beteiligungsmöglichkeiten für die Mitarbeitenden. Die Betriebe verfügen in der Regel nicht über eine übergeordnete integrative Strategie, in die sich die vereinzelten Aktionen einordnen. Die betrieblichen Alltagsroutinen sind eher nicht nachhaltig mit wenig Flexibilität für Veränderungen. Von den untersuchten Fallstudien können alle größeren Unternehmen und ein KMU diesem Typ zugeordnet werden.

Typ 4: Unternehmen mit einer experimentellen Unternehmensstrategie bieten in der Regel nur fragmentierte Unterstützungsstrukturen für nachhaltiges Alltagshandeln am Arbeitsplatz an, aber sie eröffnen den Mitarbeitenden Möglichkeiten, ihren Arbeitsplatz mitzugestalten und private Erfahrungen einzubringen. Die Hierarchien sind eher flach und die Umset-

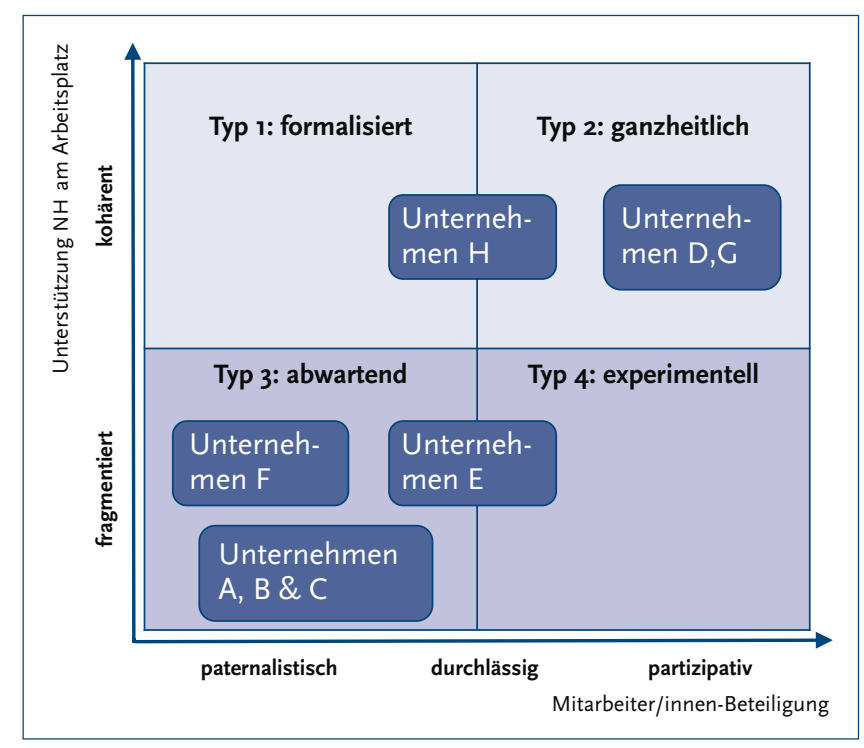

Abbildung 1: Typen von Unternehmensstrategien für greening the workplace

zung von Ideen kann individuell verfolgt werden. Diese Strategie erscheint passend für Unternehmen, die über wenige Ressourcen zur nachhaltigen Gestaltung des Arbeitsplatzes, aber über motivierte und engagierte Mitarbeitende verfügen. Es besteht allerdings das Risiko, dass sich die Beschäftigten überfordert fühlen, wenn sie keine Unterstützung in Form von zeitlichen und finanziellen Ressourcen erhalten.

\section{Schlussfolgerungen}

Die hier vorgestellte Untersuchung trägt zu Erkenntnissen zur Gestaltung nachhaltiger Arbeitsplätze als Teil einer ganzheitlichen unternehmerischen Nachhaltigkeitsstrategie bei. Nachhaltigkeit spielt in vielen alltäglichen Routinen am Arbeitsplatz, wie Ernährung, Mobilität, Umgang mit Energie und Müll, eine Rolle und kann durch das Unternehmen gezielt adressiert werden. Der von uns entwickelte Untersuchungsrahmen hat sich als hilfreich erwiesen, um die vorhandenen betrieblichen Strategien systematisch zu analysieren. Mit der Unterscheidung in die Praktikenelemente materielle und organisationale Strukturen, Bedeutung und Kompetenzen gelingt es, die Vielfalt strategischer Elemente und ihr Zusammenspiel in den Blick zu nehmen. Dabei haben sich neben formalen Regeln und Instrumenten Möglichkeiten des informellen sozialen Austauschs und Voneinander-Lernens als bedeutsam erwiesen (siehe auch Holweg et al. 2018). Ebenso ist es hilfreich sich zu vergegenwärtigen, dass es neben der Bereitstellung unterstützender Strukturen wichtig ist, neue Praktiken erproben zu können und bei ihrer dauerhaften Verankerung in alltäglichen Routinen unterstützt zu werden. Neben den aus praxistheoretischen Überlegungen abgeleiteten Kategorien hat sich die aus Ansätzen organisationalen Lernens übernommene Kategorie - die Beteiligung der Mitarbeitenden - als zentral erwiesen. 
Wir haben unterschiedliche Typen von Unterstützungsstrukturen - kohärent und fragmentiert - identifiziert. Unsere empirischen Untersuchungen zeigen, dass die Beschäftigten in Unternehmen mit kohärenten Unterstützungsstrukturen signifikant zufriedener sind. Durch die Möglichkeit, Nachhaltigkeit als gelebte Unternehmenskultur im Alltag zu erfahren, haben die Beschäftigten das Gefühl, dass das Unternehmen die eigenen Ansprüche ernst nimmt (walk the talk). Ergänzend fühlen sich Mitarbeitende in Unternehmen mit einem partizipativen Führungsstil mit ihren Ideen stärker wertgeschätzt als in solchen Betrieben mit einem eher paternalistischen Umgang. Gerade in größeren Unternehmen, die sich mit Nachhaltigkeit profilieren wollen, besteht ein großes Potenzial darin, bestehende Hürden für eine stärkere Beteiligung der Beschäftigten abzubauen.

Während einige der kleineren und mittleren „Nachhaltigkeitspioniere" nachhaltiges Alltagsverhalten am Arbeitsplatz sehr konsequent als Handlungsfeld aufgreifen, war dies bei den untersuchten größeren Unternehmen bisher deutlich seltener Fall. Selbst die durchaus vorhandenen Bemühungen werden bisher kaum nach innen und außen als Teil der betrieblichen Nachhaltigkeitsstrategie kommuniziert.

Wir schlussfolgern, dass Arbeitsplätze auf der einen Seite durch die Unternehmen gestaltet werden können (und sollten), auf der anderen Seite aber kontinuierlich durch die Mitarbeitenden mit ihrem alltäglichen Verhalten geformt werden. Der Einbezug der Bedürfnisse und Ideen der Beschäftigten kann die Kompatibilität zwischen den angebotenen Strukturen und Aktivitäten und individuellen Bedürfnissen verstärken und damit die Zufriedenheit am Arbeitsplatz erhöhen. Da es um Alltagsverhalten geht, ist es zudem ein Bereich, zu dem alle Mitarbeitenden gleichberechtigt - auch mit ihren privaten Erfahrungen - beitragen können.

\section{Literatur}

Blazejweski, S./Herbes, C. (2018): Energiewende im Unternehmen. In: ÖkologischesWirtschaften 33/1: 12-13.

Buhl, A./Blazejewski, S./Dittmer, F. (2016): The More, the Merrier. Why and how Employee-driven Eco-Innovation enhances Environmental and Competitive Advantage. In: Sustainability 8/9: 946-963.

Harris, L./Crane, A. (2002): The Greening of Organizational Culture. Management views on the Depth, Degree and Diffusion of change. In: Journal of Organizational Change Management 15/3: 214-234.

Heisserer, B. (2013): Curbing the Consumption of Distance? A PracticeTheoretical Investigation of an Employer-Based Mobility Management Initiative to Promote more Sustainable Commuting. Galway, National University of Ireland.

Holweg, C./Hermuth-Kleinschmidt, K. (2018): Eine „Denkkultur der Nachhaltigkeit" für Unternehmen. In: ÖkologischesWirtschaften 33/3: 46-50.

Klade, M./Mert, W./Seebacher, U. (2013): Sustainable Behavior at Work and in Private Life. The Contribution of Enterprises. In: International Journal of Innovation and Sustainable Development 7/4: 321-332.

Kluge, S. (2000): Empirically Grounded Construction of Types and Typologies in Qualitative Social Research. In: Forum Qualitative Sozialforschung 1/1: o.S.

Lo, S. H./Peters, G.-J./Kok, G. (2012): A Review of Determinants of and Interventions for Proenvironmental Behaviors in Organizations. In: Journal of Applied Social Psychology 42/12: 2933-2967.

Loverock, D. L. T. (2010): Employee Pro-Environmental Behaviours. Workplace Culture as a Driver for Social Change. Victoria, Royal Roads University.
Lozano, R. (2015): A Holistic Perspective on Corporate Sustainability Drivers. In: Corporate Social Responsibility and Environmental Management 22/1: 32-44.

Muster, V. (2011): Companies Promoting Sustainable Consumption of Employees. In: Journal of Consumer Policy 34/1: 161-174.

Muster, V./Schrader, U. (2011): Green Work-Life Balance. A new Perspective for Green HRM. In: Zeitschrift für Personalforschung 25/2: 140-156.

Nicolini, D. (2012): Practice Theory, Work\& Organization. An Introduction. Oxford, Oxford University Press.

Nye, M./Hargreaves, T. (2010): Exploring the Social Dynamics of Proenvironmental Behavior Change. A Comparative Study of Intervention Processes at Home and Work. In: Journal of Industrial Ecology 14/1: 137-149.

Ones, D./Dilchert, S. (2012): Environmental Sustainability at Work. A Call to Action. In: Industrial and Organizational Psychology 5/4: 444-466.

Reckwitz, A. (2002): Toward a Theory of Social Practices. A Development in Culturalist Theorizing. In: European Journal of Social Theory 5/2: 243-263.

Robertson, J. L./Barling, J. (2013): Greening Organizations through Leaders' Influence on Employees' Pro-environmental Behaviors. In: Journal of Organizational Behavior 34/2: 176-194.

Schultz, I./Seebacher, U. (2010): Sustainable Behaviour at work and in Private Life: Preconditions, Measures and Tools for Promoting a Spillover. Konferenzpapier, 6. Konferenz „Knowledge Collaboration \& Learning for Sustainable Innovation“ des ERSCP. Delft, Delft University of Technology/The Hague University of Applied Sciences/TNO.

Shove, E./Pantzar, M./Watson, M. (2012): The Dynamics of Social Practice: Everyday Life and How It Changes. London, SAGE.

Spaargaren, G./van Koppen, C. S. A. (2009): Provider Strategies and the Greening of Consumption Practices. Exploring the Role of Companies in Sustainable Consumption. In: Lange, H./Meier, L. (Hrsg.): The New Middle Classes: Globalizing Lifestyles, Consumerism and Environmental Concern. Wiesbaden, Springer: 81-100.

Süßbauer, E./Schäfer, M. (2018): Greening the Workplace. Conceptualising Workplaces as Settings for Enabling Sustainable Consumption. In: International Journal of Innovation and Sustainable Development 12/3: 327-349.

Süßbauer, E./Schäfer, M. 2019 (2019): Corporate Strategies for Greening the Workplace: Findings from Sustainability-Oriented Companies in Germany. In: Journal of Cleaner Production 226: 564-577.

Verbeek, D./Mommaas, H. (2008): Transitions to Sustainable Tourism Mobility. The Social Practices Approach. In: Journal of Sustainable Tourism 16/6: 629-644.

Warde, A. (2005): Consumption and Theories of Practice. In: Journal of Consumer Culture 5/2: 131-153.

Wolf, J. (2013): Improving the Sustainable Development of Firms. The Role of Employees. In: Business Strategy and the Environment 22/2: 92-108.

\section{AUTORINNEN + KONTAKT}

Dr. Martina Schäfer ist Professorin und wissenschaftliche Geschäftsführerin des Zentrum Technik und Gesellschaft der Technischen Universität Berlin und forscht zu Nachhaltigem Konsum, Nachhaltiger Regionalentwicklung und Methoden inter- und transdisziplinärer Forschung.

Dr. Elisabeth Süßbauer ist wissenschaftliche Mitarbeiterin am Zentrum Technik und Gesellschaft der Technischen Universität Berlin und forscht zu Nachhaltigem Konsum. Seit Juni 2019 leitet sie die Nachwuchsgruppe Mit Precycling zu mehr Ressourceneffizienz (PuR).

Zentrum Technik und Gesellschaft der Technischen Universität Berlin, Sekr. HBS 1, Hardenbergstr. 16-18, 10623 Berlin. Tel.: +49 3031426854 , E-Mail: schaefer@ztg.tu-berlin.de, suessbauer@ztg.tu-berlin.de, Website: https://www.tu-berlin.de/ztg/menue/
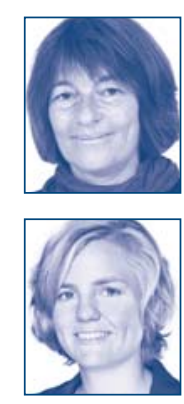
startseite_ztg/ 